\title{
Binding of biexcitons in GaAs/AlxGa1-xAs superlattices
}

Mizeikis, Vygantas; Birkedal, Dan; Langbein, Wolfgang Werner; Vadim, Lyssenko; Hvam, Jørn Märcher

Published in:

Physical Review B

Link to article, DOI:

10.1103/PhysRevB.55.5284

Publication date:

1997

Document Version

Publisher's PDF, also known as Version of record

Link back to DTU Orbit

Citation (APA):

Mizeikis, V., Birkedal, D., Langbein, W. W., Vadim, L., \& Hvam, J. M. (1997). Binding of biexcitons in GaAs/AlxGa1-xAs superlattices. Physical Review B, 55(8), 5284-5289.

https://doi.org/10.1103/PhysRevB.55.5284

\section{General rights}

Copyright and moral rights for the publications made accessible in the public portal are retained by the authors and/or other copyright owners and it is a condition of accessing publications that users recognise and abide by the legal requirements associated with these rights.

- Users may download and print one copy of any publication from the public portal for the purpose of private study or research.

- You may not further distribute the material or use it for any profit-making activity or commercial gain

- You may freely distribute the URL identifying the publication in the public portal 


\title{
Binding of biexcitons in $\mathrm{GaAs} / \mathrm{Al}_{x} \mathrm{Ga}_{1-x} \mathrm{As}$ superlattices
}

\author{
V. Mizeikis, ${ }^{*}$ D. Birkedal, W. Langebein, V. G. Lyssenko, ${ }^{\dagger}$ and J. M. Hvam \\ Mikroelektronik Centret, The Technical University of Denmark, DK-2800 Lyngby, Denmark
}

(Received 28 May 1996)

\begin{abstract}
Properties of the heavy-hole excitons and biexcitons in $\mathrm{GaAs} / \mathrm{Al}_{0.3} \mathrm{Ga}_{0.7} \mathrm{As}$ superlattices are studied using linear and nonlinear optical techniques. In superlattices with miniband halfwidths less than the exciton binding energy, the biexciton binding energy is found to be the same as in the noninteracting multiple quantum wells of the same width. When the miniband halfwidth exceeds the exciton binding energy, the biexciton binding energy decreases abruptly to the bulk value. [S0163-1829(97)02008-0]
\end{abstract}

\section{INTRODUCTION}

The binding energies of excitons and biexcitons in $\mathrm{GaAs} / \mathrm{Al}_{x} \mathrm{Ga}_{1-x} \mathrm{As}$ quantum wells are significantly enhanced compared to bulk GaAs. The main reason for this enhancement is the one-dimensional confinement of the electrons and holes. If the confinement becomes weaker, for instance, when the quantum well width increases, exciton and biexciton binding energies decrease, finally approaching bulk values.

It was predicted recently that in two dimensions, the relation between the biexciton and exciton binding energies $E_{x x}^{b}$ and $E_{x}^{b}$ can be expressed as ${ }^{1}$

$$
E_{x x}^{b} / E_{x}^{b} \approx 0.228
$$

This result was confirmed experimentally for the heavy-hole (HH) excitons and biexcitons in the quantum wells with thicknesses in the range of $80-160 \AA{ }^{2}$ Hence both quantities scale similarly when the well width is varied, indicating that a two-dimensional description applied to quantum wells of finite thickness yields good agreement with theory. In superlattices (SL's), it is possible to change the confinement by varying the thickness of the $\mathrm{Al}_{x} \mathrm{Ga}_{1-x} \mathrm{As}$ barriers, while maintaining the GaAs well thickness constant. The interwell coupling, which gives rise to energy minibands and delocalization of states in the superlattice growth direction, is controlled by the barrier thickness. Its gradual decrease will result in the transition from isolated quantum wells to the superlattice and ultimately to the bulk solid. The delocalization of the excitons and the accompanying change of the exciton binding energy during this transition has been studied earlier. ${ }^{3,4}$ The behavior of biexcitons and their binding energy has not been addressed so far, and in this paper we experimentally investigate this problem.

In the limit of thick barriers, i.e., in the multiple-quantumwell (MQW) structure, the biexciton binding energy is proportional to the exciton binding energy according to the relation (1). In the other limiting case of vanishing barrier thickness, the material properties approach these of the bulk GaAs and Eq. (1) is transformed into Haynes rule for threedimensional (3D) biexcitons: $:^{5,6}$

$$
E_{x x}^{b} / E_{x}^{b} \approx 0.1
$$

which again establishes the proportionality between the two binding energies. In an attempt to study the transition between the two regimes, we have measured the heavy-hole exciton and biexciton binding energies as a function of barrier width for a fixed well width. The exciton binding energies were obtained from linear optical measurements and calculations, while the biexciton binding energies were measured from exciton-biexciton quantum beats in transient four-wave-mixing (TFWM) experiments. ${ }^{7}$ We show that the biexciton binding energy remains constant when the superlattice barriers become thinner, until an abrupt decrease to the bulk value that occurs at a certain critical barrier thickness. This sharp change in the binding energy is associated with the transition from quasi-two-dimensional biexcitons to bulk biexcitons and occurs when the superlattice miniband halfwidth exceeds the exciton binding energy in the isolated quantum well of the same thickness. The decrease in the exciton binding energy is smoother than that of the biexciton; hence, in narrow-miniband superlattices the two binding energies are not proportionally related to each other.

\section{EXPERIMENTAL DETAILS}

The samples investigated in this work are designed with the aim of studying the transition from the MQW to the SL structure. Five heterostructures, grown by molecular-beam epitaxy on (100) semi-insulating GaAs substrates, consist of 20 periods of $\mathrm{GaAs} / \mathrm{Al}_{0.3} \mathrm{Ga}_{0.7} \mathrm{As}$. The thicknesses of the GaAs quantum wells are the same $\left(L_{w}=80 \AA\right)$ in all samples, while the thicknesses of the $\mathrm{Al}_{x} \mathrm{Ga}_{1-x}$ As barriers are different: $L_{b}=200,100,50,30$, and $20 \AA$. In the following we refer to the samples by their barrier thickness. To allow for the linear transmission measurements and TFWM experiments in transmission geometry, the samples were lifted off the GaAs substrates and mounted on sapphire disks. Characterization by $\mathrm{cw}$ photoluminescence and photoluminescence-excitation (PLE) techniques was performed on the samples before the substrate removal. The inhomogeneous linewidth of the heavy-hole exciton line was about $2.2 \mathrm{meV}$ full width at half maximum in all samples, except the one with $L_{b}=20 \AA$, which had a smaller linewidth of $1.5 \mathrm{meV}$. The Stokes shifts in all samples varied within the range $0.4-0.7 \mathrm{meV}$. All characterizations and experiments were carried out at a temperature of $4 \mathrm{~K}$, which was maintained in a helium bath cryostat. 
Transient four-wave-mixing experiments are performed using femtosecond pulses from a $76-\mathrm{MHz}$ repetition rate Ti:sapphire laser. The duration of the transform-limited pulses is $120 \mathrm{fs}$ and their central frequency is tuned to excite excitonic and biexcitonic resonances simultaneously. The two-beam self-diffraction geometry ${ }^{8}$ and time-integrated signal detection are used in the TFWM experiments. The signal is recorded, as a function of the delay $t_{12}$ between the two coherent laser pulses, in the nearly phase-matched direction $2 \mathbf{k}_{2}-\mathbf{k}_{1}$, where $\mathbf{k}_{1}$ and $\mathbf{k}_{2}$ are the wave vectors of the incident pulses. The decay of the TFWM signal $I_{\mathrm{TFWM}}$ can, for a three-level system, be expressed as ${ }^{9}$

$$
I_{\mathrm{TFWM}}\left(t_{12}\right) \propto \exp \left(-\frac{c t_{12}}{T_{2}}\right)\left[1+I_{m}\left(t_{12}\right) \cos \left(\frac{E_{1}-E_{2}}{\hbar} t_{12}\right)\right],
$$

where the constant $c=2$ (4) for homogeneously (inhomogeneously) broadened systems and $T_{2}$ is the phenomenological dephasing time. The expression in the square brackets accounts for the quantum beats. $E_{1}$ and $E_{2}$ are the energies of the beating resonances and $I_{m}\left(t_{12}\right)$ denotes the delaydependent amplitude of the signal modulation. The energy difference $E_{1}-E_{2}$ can be determined from the oscillation period in $I_{\mathrm{TFWM}}\left(t_{12}\right)$. To obtain the maximum available information in the TFWM experiment, ${ }^{9}$ the signal is dispersed in the spectrometer and its spectrum is recorded using the optical multichannel analyzer system as a slow detector.

\section{RESULTS AND DISCUSSION}

The energy of the exciton in the ground state can be expressed as $E_{x}=E_{g}^{\prime}-E_{x}^{b}$, where $E_{g}^{\prime}$ is the energy gap in the material, modified by the confinement. The biexciton energy is $E_{x x}=2 E_{x}-E_{x x}^{b}$. The exciton binding energy can be determined from the linear absorption spectrum as the difference between the $1 s$ peak and the onset of the band-to-band continuum transitions according to the well-known Elliott formula. ${ }^{10,11}$ Due to their inherently nonlinear nature, biexcitonic effects become observable in the nonlinear regime, i.e., at high $e-h$ pair densities. With resonant excitation, biexcitons can be created via two-photon transitions with a single exciton as an intermediate state. ${ }^{12} E_{b}^{x x}$ in this case can be determined from the energy difference between the biexciton and exciton resonance enhancements in the TFWM spectrum, representing the third-order nonlinear susceptibility, or from the period of quantum beats in the TFWM signal decay. ${ }^{7}$

The spectra of the linear optical density of all samples are shown in Fig. 1. For better comparison, the individual spectra are shifted an amount $\Delta$ along the energy axis in order to make the HH exciton peaks in all plots coincident. The absolute energies of the heavy-hole and light-hole (LH) excitonic peaks vary somewhat from sample to sample. The likely reason for this is that thin, optically transparent samples mounted on the sapphire disks experience accidental mechanical strains. The amplitude of these random shifts is within a few meV range, and in Fig. 1 they are compensated by the horizontal offsets. As can be seen from Fig. 1, the HH-LH splitting increases with the decrease in the barrier width from 200 to $50 \AA$, and this anomalous behavior can be

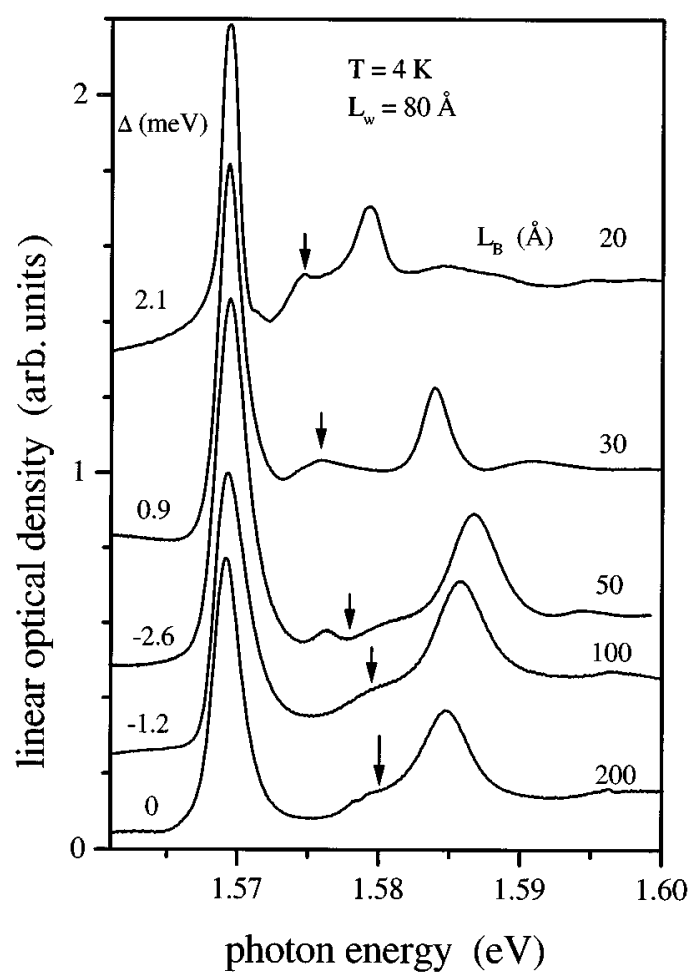

FIG. 1. Spectra of linear optical density in $\mathrm{GaAs} / \mathrm{Al}_{x} \mathrm{Ga}_{1-x} \mathrm{As}$ quantum wells and superlattices. The onset of the $\mathrm{HH}$ continuum transitions, corresponding to the calculated values of $E_{x}^{b}$ (see Table I), is marked by the arrows. $\Delta$ indicates the horizontal offset for each spectrum.

also attributed to the influence of strain.

In spite of these undesirable variations, the spectra in Fig. 1 show obvious signatures of the superlattice formation with decreasing barrier width. The samples with thickest barriers ( $L_{b}=200$ and $100 \AA$ ) almost exactly correspond to the case of the isolated quantum wells. The HH continuum is seen as a relatively weak shoulder 9-12 $\mathrm{meV}$ above the $\mathrm{HH}$ exciton resonance. The direct determination of the exciton binding energy from the spectra in Fig. 1 is difficult, but in these two samples it was possible to detect a pronounced HH continuum edge in the PLE spectra (not shown here). From these spectra $E_{x}^{b} \approx 11 \mathrm{meV}$ is estimated. For the samples with $L_{b} \leqslant 50 \AA$, the $\mathrm{HH}$ continuum edge becomes more pronounced and gradually approaches the HH exciton peak when the barrier thickness is decreased. In the 20- $\AA$ sample $E_{x}^{b} \approx 5 \mathrm{meV}$ can be directly determined from the absorption spectrum. Another identifying characteristic of the superlattice effect is the interwell exciton, which forms due to the Coulomb coupling and state overlap between adjacent quantum wells. It is clearly resolved in the spectrum for the $50-\AA$ sample as a peak around $1.579 \mathrm{eV}$. In the $30-$ and $20-\AA$ samples, it is partially overlapping with the HH continuum edge. Properties of the interwell excitons have been reported earlier. $^{13}$

As was mentioned before, the HH-LH splitting is influenced by the strain and fluctuates within a $2-\mathrm{meV}$ range in $200-, 100-$, and $50-\AA$ samples. However, in the $20-\AA$ sample the splitting becomes so significantly reduced (by $\approx 6 \mathrm{meV}$ ) that it can no longer be attributed to the strain or other side effects. It is an apparent indication that the confinement, 
TABLE I. Parameters of GaAs/ $\mathrm{Al}_{x} \mathrm{Ga}_{1-x} \mathrm{As}$ superlattices.

\begin{tabular}{llccccccc}
\hline \hline$L_{b}(\AA)$ & $\mu_{z e}^{*}$ & $\Delta E_{e}(\mathrm{meV})$ & $\mu_{z \mathrm{HH}}^{*}$ & $\Delta E_{\mathrm{HH}}(\mathrm{meV})$ & $\mu_{z e}^{*(3 \mathrm{D}) a}$ & $\mu_{z \mathrm{HH}}^{*(3 \mathrm{D}) a}$ & $\alpha$ & $E_{x}^{b}(\mathrm{meV})$ \\
\hline 200 & $\rightarrow \infty$ & $\rightarrow 0$ & $\rightarrow \infty$ & $\rightarrow 0$ & 0.075 & 0.46 & 2.24 \\
100 & 1.5 & 0.14 & $\rightarrow \infty$ & $\rightarrow 0$ & 0.08 & 0.43 & 2.27 \\
50 & 0.3 & 3.3 & 17.6 & $\rightarrow 0$ & 0.072 & 0.4 & 2.39 \\
30 & 0.13 & 12 & 1.65 & 0.8 & 0.07 & 0.38 & 2.61 \\
20 & 0.094 & 23.5 & 0.8 & 2.3 & 0.07 & 0.341 & 2.76 \\
\hline \hline
\end{tabular}

${ }^{a}$ According to Ref. 18 .

which lifts the degeneracy between the heavy and light holes, is reduced too. In the same sample, the heavy-hole exciton line is much narrower than the other ones. This feature can be explained as a consequence of the increased exciton volume. The exciton, delocalized in the $z$-axis direction, spreads over several SL periods and becomes insensitive to local well width and alloy fluctuations, ${ }^{3}$ which are the main source of inhomogeneous broadening in the quantum wells.

\section{A. Exciton binding}

To demonstrate further that the observed evolution of the linear optical properties with decreasing $L_{b}$ is not an artifact (e.g., due to the accidental strains), but a true manifestation of the superlattice formation, we have calculated the exciton binding energies in these structures using the fractionaldimensional approach. ${ }^{14}$ This approach provides a relatively easy way to obtain the exciton binding energies in the systems, which are neither purely two dimensional nor three dimensional. A noninteger dimensionality parameter $\alpha$ can be used to describe the state "compression" in the anisotropic system. Integer values of $\alpha=1,2,3$ correspond to the common one-, two-, and three-dimensional cases. The exciton binding energy depends on $\alpha$ as ${ }^{15}$

$$
E_{x}^{b}=E_{0}\left[\frac{2}{\alpha-1}\right]^{2},
$$

where $E_{0}$ is the bulk exciton binding energy $(\approx 4.2 \mathrm{meV}$ at low temperatures for GaAs). In quantum wells and superlattices $\alpha$ can be approximated by ${ }^{15}$

$$
\alpha=3-\left[1-\frac{\mu_{0 z}^{*(3 \mathrm{D})}}{\mu_{z}^{*}}\right] \exp \left[-\frac{L_{w}^{*}}{2 a_{0}}\right] .
$$

Here $\mu_{z}^{*}$ and $\mu_{0 z}^{*(3 D)}$ are the reduced masses along the $z$-axis direction in the periodic superlattice and in the equivalent bulk alloy, respectively, $L_{w}^{*}$ is the "effective width", 15 of the quantum well, and $a_{0}$ is the $3 \mathrm{D}$ excitonic Bohr radius. To obtain the required quantities, we have calculated the band structures of all samples using the $\mathbf{k} \cdot \mathbf{p}$ method. ${ }^{16,17}$ The parameters used in the calculations are $L_{b}, L_{w}$, and the band offsets between the well and barrier materials. Results relevant for this work are the electron and heavy-hole miniband widths and effective masses. They are denoted $\Delta E_{e}$, $\Delta E_{\mathrm{HH}}, \mu_{z e}^{*}$, and $\mu_{z \mathrm{HH}}^{*}$ and are listed in Table I. The effective width $L_{w}^{*}$ has been adjusted to match the experimental value of the exciton binding energy of the $200-\AA$ sample. To obtain $\mu_{0 z}^{*(3 \mathrm{D})}$ in the bulk alloy with the same aluminium contents as in the superlattice, the relevant electron and heavy-hole effective masses are taken from the literature. ${ }^{18}$ They are given in Table I along with the calculated values of $\alpha$ and $E_{x}^{b}$.

The results of the calculations in Table I are consistent with the spectral signatures of the superlattice formation, presented in Fig. 1. The absence of dispersion in the samples with $L_{b}=200$ and $100 \AA$ confirms their MQW properties. In structures with $50-\AA$ and thinner barriers, the superlattice properties begin to evolve gradually, mainly due to the electronic miniband formation. The electron effective mass decreases with the barrier width and becomes comparable to the effective mass in the $x-y$ plane in 30- and 20- $\AA$ samples. The heavy-hole states are less affected by the superlattice formation, as can be seen from their large effective masses and narrow minibands. Only in the $20-\AA$ sample, the heavyhole miniband acquires a non-negligible width of $2.3 \mathrm{meV}$. The anisotropy of the heavy-hole excitonic states decreases with $L_{b}$, as evidenced by the increase in the fractional dimension from 2.24 to 2.76 . Correspondingly, the heavy-hole exciton binding energy decreases from 11 to $5.4 \mathrm{meV}$.

In Fig. 1, the heavy-hole continuum edge is clearly expressed only in the spectrum for $L_{b}=20 \AA$. For this sample, $E_{x}^{b} \approx 5.2 \mathrm{meV}$ can be directly determined, in good agreement with the calculation. For the 50- and 30- $\AA$ samples, PLE measurements with adequate precision were not possible due to their lower luminescence efficiency. This is caused by the spread of the electronic states into the $\mathrm{Al}_{x} \mathrm{Ga}_{1-x} \mathrm{As}$ barriers increasing the efficiency of nonradiative recombination processes. A precise evaluation of $E_{x}^{b}$ in these samples from the spectra in the Fig. 1 is difficult. However, the calculated exciton binding energies seems to be in general agreement with the observed absorption line shapes. The onset of the $\mathrm{HH}$ continuum edge, corresponding to the calculated $E_{x}^{b}$, indicated in Fig. 1 by the arrows. Therefore, we rely on the calculated binding energies for these two samples.

\section{B. Biexciton binding}

The binding energies of the heavy-hole biexcitons are measured in the transient four-wave-mixing experiments. The biexciton binding is enhanced in MQWs and $E_{x x}^{b}$ varies within the range 1.0-2.6 meV, depending on the well width and degree of localization. ${ }^{2,7,12,19}$ When the quantum well width varies from 80 to $160 \AA$, the ratio between the biexciton and exciton binding energies has been found to be constant ${ }^{2}$ as described by Eq. (1). The fractional dimension, which was determined for the superlattices earlier in this 


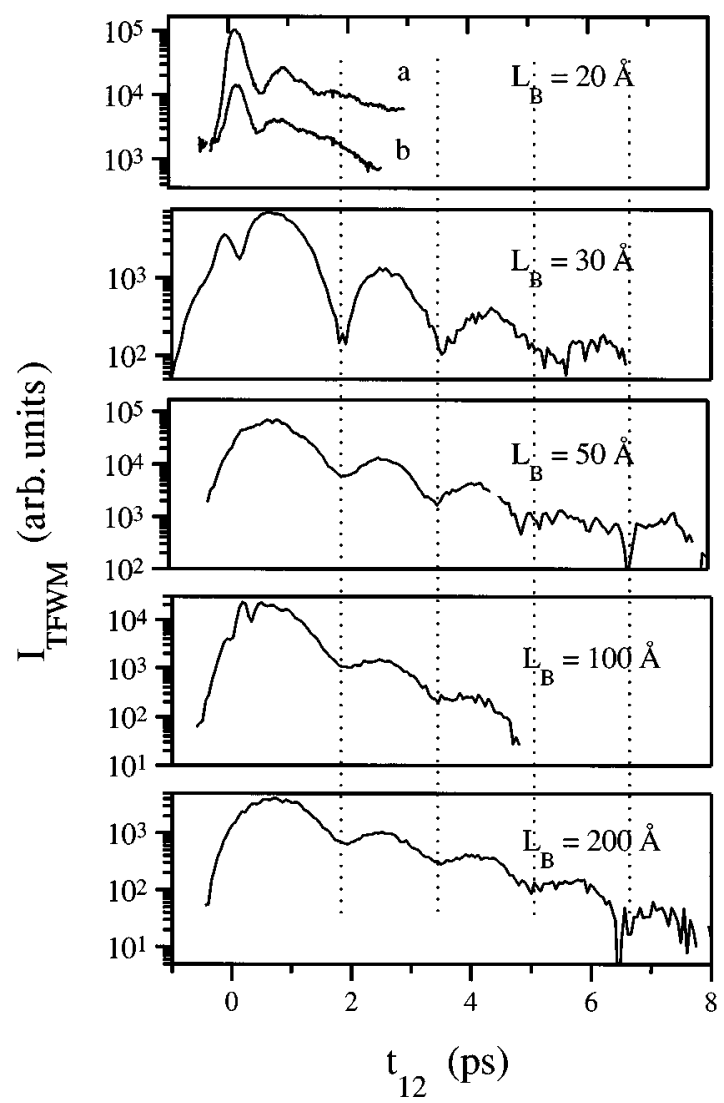

FIG. 2. Decays of the TFWM signal as a function of delay $t_{12}$ at the spectral position of the heavy-hole biexciton in samples with different barrier width. Vertical dashed lines are guides to the eye marking the signal minima. In the top plot, the signal decays in the $20-\AA$ sample, measured (a) at the $\mathrm{HH}$ exciton energy and (b) 2.5 meV below it, are shown.

work, is a very general characteristic of the anisotropy in the system. The change in the fractional dimension, accompanying the change in the well width from 80 to $160 \AA$, can be roughly estimated using Eq. (5) and is in the range 2.35-2.5. As can be seen from the Table I, we have, in the present study, the possibility to change the anisotropy in an even broader range by changing the barrier width and observe the corresponding behavior of the biexciton binding energy.

Nonlinear quantum beat spectroscopy provides a straightforward way to determine the biexciton binding energy from the period of exciton-biexciton beats in the TFWM signal. ${ }^{7}$ In samples where inhomogeneous broadening of the $\mathrm{HH}$ exciton is similar to the biexciton binding energy, the modulation of the spectrally integrated TFWM signal due to quantum beats is somewhat suppressed; however, pronounced beating can still be observed if the TFWM signal is spectrally resolved and detected at the energy of the biexcitonic resonant enhancement. In Fig. 2 the TFWM signal is shown, measured about $2.5 \mathrm{meV}$ below the energy of the excitonic resonant enhancement in all the investigated samples. The central frequency of the laser pulse is tuned about $2-3 \mathrm{meV}$ below the exciton in order to reduce the influence of continuum excitation.

As seen from Fig. 2, the signal decay reveals pronounced oscillations, which we attribute to the exciton-biexciton quantum beats in all samples except the one with narrowest

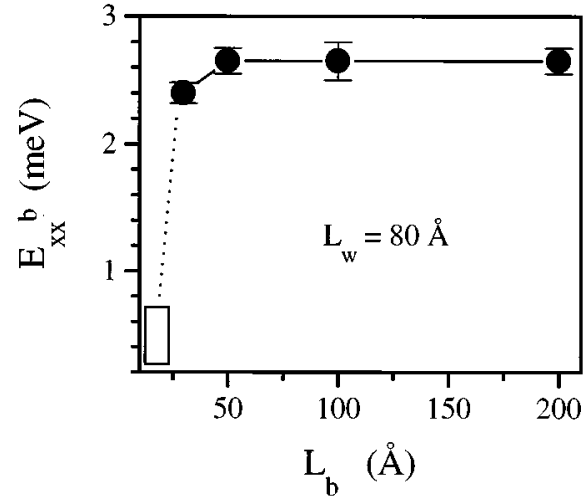

FIG. 3. Heavy-hole biexciton binding energy as a function of barrier width. The dotted line and $\square$ show the expected behavior of the biexciton binding energy due to the $2 \mathrm{D}$ bulk transition and its value in the 20 - $\AA$ sample.

barriers $\left(L_{b}=20 \AA\right)$. The period of the beat is about $1.6 \mathrm{ps}$, which corresponds to a $2.6-\mathrm{meV}$ difference in energy. Furthermore, the beat period is nearly the same in all plots, indicating that the biexciton binding energy is essentially the same in all samples. This is not surprising in the structures with $L_{b}=200$ and $100 \AA$ because they are almost identical MQW structures. However, in the 50- and $30-\AA$ samples, where the lower exciton binding energies indicate their partial delocalization along the $z$ axis, the biexciton binding energy remains essentially the same as in the isolated quantum wells. This point is further emphasized in Fig. 3, where $E_{x x}^{b}$ is plotted as a function of the barrier width.

In the sample with narrowest barriers $\left(L_{b}=20 \AA\right)$, no beat signal was detected below the HH exciton. There can be several reasons for this. First, the HH exciton is closer to the heavy- and light-hole continuum edge in this sample. As a result, more states belonging to both continua are excited with the spectrally broad laser. A variety of effects due to the continuum excitation have been reported. Without going into details, we note that they lead either to faster dephasing and resonance broadening due to the exciton-free carrier collisions, ${ }^{20}$ or to quenching of the TFWM signal for delays, exceeding the temporal width of the laser pulse. ${ }^{21,22}$ Both effects will impede the experimental observation of the quantum beats.

However, the most likely reason for the suppression of the beats is the drastically reduced biexciton binding energy in this sample. We suggest that at a certain critical barrier thickness $20 \AA<L_{b}<30 \AA$, biexcitons lose their quasi-twodimensional character and spread in the $z$-axis direction with an accompanying decrease in their binding energy. For the bulk biexciton, the 3D Hayne rule (2) applies and its binding energy $(\leqslant 0.5 \mathrm{meV})$ is very low compared to the twodimensional case. The period of the quantum beat, which is inversely proportional to the energy splitting, will become longer than the dephasing time, making it impossible to observe the beating. As can be seen from Fig. 3, the biexciton binding energy is slightly smaller in the $30-\AA$ sample, and this feature could mark the onset of the abrupt change. In the same figure, we also indicate the anticipated behavior of $E_{x x}^{b}$ when superlattice barriers decrease from 30 to $20 \AA$. 
The results for samples with 200- and 100- $\AA$ barriers can be directly compared with previous findings in quantum wells. We obtain $E_{x x}^{b} / E_{x}^{b} \approx 0.24$, taking the measured value $(\approx 11 \mathrm{meV})$ of the exciton binding energy. This result is in good agreement with previously reported ${ }^{2} E_{x x}^{b} / E_{x}^{b} \approx 0.2$. In spite of the consistency at this starting point, the ratio begins to deviate significantly from the typical quantum-well value when going to narrower barriers. For the $L_{b}=50$ and $30 \AA$ samples, taking the calculated exciton binding energies from Table I, the ratio (1) increases to 0.30 and 0.40 due to the decrease in the exciton binding energy. As one can see from Table I and Fig. 1, a gradual reduction of the exciton binding energy can be associated with the spreading of this state along the superlattice growth direction. With respect to the periodic confining potential, quantum-well excitons are Frenkel-like states because they are localized within the single elementary cell. Correspondingly, delocalized excitons in the superlattices are Wannier-like states. ${ }^{15}$ From our data it seems that for the excitons the transition from Frenkel-like to Wannier-like regimes is quite smooth, while the biexcitons exhibit an almost steplike transition. Consequently, in 50- and 30- $\AA$ samples we have an openly conflicting situation, where a Frenkel-like biexciton is formed by the interaction between two Wannier-like excitons.

This can be solved by realizing that, in fact, the $n=1$ excitons are Frenkel-like states, just like biexcitons, in all investigated samples, except the one with $L_{b}=20 \AA$ A. In these structures both excitons and biexcitons are localized within the single quantum well. Such localization can be explained in terms of the Coulomb interaction between the electron and heavy hole. It was shown theoretically and experimentally that in the superlattices with the miniband halfwidth less than the exciton binding energy (in single quantum well of the same thickness), electrons are localized by the Coulomb potential of relatively immobile heavy holes. As a result, the $n=1 \mathrm{HH}$ exciton states are confined inside single quantum wells and their penetration to the neighboring wells is reduced. Such localization was found to be responsible for the suppression of Stark ladder transitions in biased superlattices ${ }^{23}$ and the formation of the interwell exciton. ${ }^{2}$ It is clear from the data in Table I that all investigated samples, except the one with $L_{b}=20 \AA$, satisfy the condition

$$
\Delta E / 2<E_{x}^{b, \mathrm{MQW}},
$$

where $\Delta E$ is the combined width of the electron and heavyhole minibands and $E_{x}^{b, \mathrm{MQW}} \approx 11 \mathrm{meV}$ is the exciton binding energy in 200- $\AA$ sample. Relation (6) compares the effect of the periodic superlattice potential to that of the photoexcited single heavy hole. In narrow miniband superlattices satisfying Eq. (6), single electronic states are extended in the $z$-axis direction. For the electron interacting with the heavy hole in the same quantum well, the translational symmetry of the superlattice is destroyed by the heavy-hole potential and the resulting pair state is localized along the $z$ axis. ${ }^{23,24}$ The same is true for the biexciton because in this case the electron energy is lowered even more. In practice this situation corresponds to all samples, except the one with $L_{b}=20 \AA$. As the superlattice barrier width decreases from 30 to $20 \AA$, condition (6) is violated and the hole potential cannot localize the electrons along the $z$ axis. Heavy-hole excitons and biexcitons will spread in space over several superlattice periods. In this regime both excitons and free carriers have three-dimensional degrees of freedom and even the heavyhole mobility along the $z$ axis is higher than in other samples (see Table I).

We tentatively explain the abrupt nature of the transition from the two-dimensional to bulk behavior as follows. It has been shown that in two dimensions it is possible to transform the Hamiltonian of a biexciton into that of a single exciton with modified parameters. ${ }^{1}$ The validity of this model for $80-160 \AA$ quantum wells has been proven experimentally. ${ }^{2}$ Treating the biexcitons as excitons, it is relevant to refer to the previous studies of the excitons in the superlattices undergoing transition from two to three dimensions. Variational calculations ${ }^{3}$ show that even though the decrease in the exciton binding energy is fairly smooth, the exciton radius in the $z$-axis direction increases abruptly when the superlattice period $L_{b}+L_{w}$ reaches a critical value. In our case of biexcitons this means that at a critical barrier thickness (supposedly $20<L_{b}<30 \AA$ ), the two-dimensional model of biexciton becomes irrelevant and we observe abrupt transition to the bulk state.

\section{CONCLUSION}

We have studied the biexciton binding in GaAs/ $/ \mathrm{Al}_{x} \mathrm{Ga}_{1-x}$ As superlattices with different barrier thicknesses and miniband widths. For the superlattices with miniband halfwidths smaller than the exciton binding energy, we find a high biexciton binding energy of $2.6 \mathrm{meV}$, which is nearly independent of the superlattice barrier thickness. At the same time, the exciton binding energy smoothly decreases with the barrier thickness. We find that the biexcitonic states in the superlattices change from quasi-twodimensional to bulk character in a steplike manner when the barrier thickness becomes critically low. These features are explained in terms of the localization, mediated by the Coulomb interaction in the narrow miniband superlattices.

\section{ACKNOWLEDGMENTS}

This work was supported by the Danish Ministries of Industry and Research in the framework of CNAST. All the samples used were grown by C.B. Sørensen.
*Permanent address: Department of Solid State Electronics, Vilnius University, Sauletekio Avenue 9, Building III, LT-2054 Vilnius, Lithuania.

†Permanent address: Institute of Microelectronics Technology and Superpure Materials, Chernogolovka, Moscow District, 142432, Russia.
${ }^{1}$ J. Singh, D. Birkedal, V. Lyssenko, and J. Hvam, Phys. Rev. B 45, 15909 (1996).

${ }^{2}$ D. Birkedal et al., Phys. Rev. Lett. 76, 672 (1996).

${ }^{3}$ A. Chomette et al., Europhys. Lett. 4, 461 (1987).

${ }^{4}$ M. F. Pereira, I. Galbraith, S. Koch, and G. Duggan, Phys. Rev. B 42, 7084 (1990). 
${ }^{5}$ J. Haynes, Phys. Rev. Lett. 4, 361 (1960).

${ }^{6}$ J. Haynes, Phys. Rev. Lett. 17, 860 (1966).

${ }^{7}$ K.-H. Pantke et al., Phys. Rev. B 47, 2413 (1993).

${ }^{8}$ L. Schultheis, M. D. Sturge, and J. Hegarty, Appl. Phys. Lett. 47, 995 (1985).

${ }^{9}$ J. Erland et al., Phys. Rev. B 50, 15047 (1994).

${ }^{10}$ R. J. Elliott, Phys. Rev. 108, 1384 (1957).

${ }^{11} \mathrm{H}$. Haug and S. Koch, Quantum Theory of the Optical and Electronic Properties of Semiconductors (World Scientific, Singapore, 1993).

${ }^{12}$ D. J. Lovering, R. T. Philips, G. J. Denton, and G. W. Smith, Phys. Rev. Lett. 68, 1880 (1992).

${ }^{13}$ D. Birkedal et al., Nuovo Cimento 17D, 1359 (1995); Phys. Rev. B 54, 10316 (1996).

${ }^{14}$ X.-F. He, Phys. Rev. B 43, 2063 (1991).
${ }^{15}$ P. Lefebvre, P. Christol, and H. Mathieu, Phys. Rev. B 46, 13603 (1992).

${ }^{16}$ J. Luttinger, Phys. Rev. B 102, 1030 (1956).

${ }^{17}$ P. Y. Yu and M. Cardona, Fundamentals of Semiconductors (Springer-Verlag, Berlin, 1996).

${ }^{18}$ Properties of Aluminium Gallium Arsenide, EMIS Datareviews No. 7, edited by S. Adachi (INSPEC, London, 1993).

${ }^{19}$ D. A. Kleinman, Phys. Rev. B 28, 871 (1983).

${ }^{20}$ A. Honold, L. Schultheis, J. Kuhl, and C. W. Tu, Phys. Rev. B 40, 6442 (1989).

${ }^{21}$ J. Feldmann et al., Phys. Rev. Lett. 70, 3027 (1993).

${ }^{22}$ D. Birkedal, V. Lyssenko, J. Hvam, and K. E. Sayed, Phys. Rev. B 54, 14250 (1996).

${ }^{23}$ A. Fox et al., Phys. Rev. B 46, 15365 (1992).

${ }^{24}$ G. Cohen, I. Bar-Joseph, and H. Shtrikman, Phys. Rev. B 50, 17316 (1994). 